\title{
Immune pathogenesis of COVID-19-related multisystem inflammatory syndrome in children
}

\author{
Anne H. Rowley, ${ }^{1,2}$ Stanford T. Shulman, ${ }^{1}$ and Moshe Arditi ${ }^{3,4}$ \\ 'Department of Pediatrics and 2Department of Microbiology/Immunology, Northwestern University Feinberg School of Medicine and Ann \& Robert H. Lurie Children's Hospital of Chicago, Chicago, Illinois, \\ USA. ${ }^{3}$ Department of Pediatrics, Division of Pediatric Infectious Diseases and Immunology, and ${ }^{4}$ Department of Biomedical Sciences, Infectious and Immunologic Diseases Research Center (IIDRC), \\ Cedars-Sinai Medical Center, Los Angeles, California, USA.
}

\section{An emerging understanding of MIS-C}

When cases of severe acute respiratory syndrome coronavirus 2-associated (SARSCoV-2-associated) multisystem inflammatory syndrome in children (MIS-C) were initially reported from Europe, the predominant clinical findings were persistent fever, marked abdominal symptoms, cytokine storm, myocardial dysfunction, and cardiogenic shock with left ventricular dysfunction in the setting of multisystem inflammation, reminiscent of toxic shock syndrome (TSS) or Kawasaki disease shock syndrome (KDSS) and requiring ICU care (1). Other clinical findings included those commonly observed in children with a variety of different infections and noninfectious illnesses, such as conjunctival injection, oral mucosal changes, and rash, features that are overlapping with TSS and incomplete Kawasaki disease. Because mucocutaneous findings are present in children with Kawasaki disease, and because patients with MIS-C sometimes developed mild coronary artery dilation, diagnostic confusion initially led some clinicians to conclude that the two conditions were the same (2).

\section{Distinctions from Kawasaki disease}

There are several important features of MIS-C that differentiate it from Kawasaki disease (3). Recent data indicate that coronary artery dilation in patients with MIS-C is mild and transient (4), similar to that observed in some other febrile illnesses of childhood, including systemic onset juvenile idiopathic arthritis (5). No coronary artery inflammation or necrotiz- ing arteritis has been observed in autopsy studies in adults with coronavirus 2019 (COVID-19) $(6,7)$, and although data on patients with MIS-C are limited, a recent case report of an 11-year-old patient described myocarditis in the absence of coronary artery inflammation (8).

It is too early to define the pathological mechanism of coronary artery dilation seen in MIS-C with certainty, but a likely contributor is the endothelial dysfunction (without morphological changes) associated with SARS-CoV-2 infection, which is sustained by the cytokine storm. This proposed mechanism may explain the less severe and more transient coronary artery dilation in patients with MIS-C compared with those with Kawasaki disease. The transient nature of coronary artery findings in MIS-C are in marked contrast to the more severe dilation and coronary artery aneurysm formation observed in Kawasaki disease, which can lead to myocardial infarction, aneurysm rupture, and sudden death (9).

Another important difference between the diseases is in the epidemiology; the highest attack rate and most severe sequelae of Kawasaki disease occur in infants (10-12), whereas the median age of MIS-C appears to be about 9 years of age, with infants relatively spared (13). Moreover, children of Asian descent experience the highest attack rates of Kawasaki disease in the world (10), but no cases of MIS-C have been reported from Asia $(14,15)$. Finally, marked lymphopenia is a common laboratory finding in MIS-C (13), but it is not a feature of Kawasaki disease. A recent study by Carter et al. reported immunophenotyping of patients with MIS-C from the United
Conflict of interest: AHR and STS are named investigators on provisional patent 62/811,930, "Antibodies and antigens of Kawasaki disease."

Copyright: () 2020, American Society for Clinical Investigation.

Reference information: / Clin Invest. 2020;130(11):5619-5621. https://doi.org/10.1172/JCI143840.
Kingdom and concluded that MIS-C presents as an immunopathogenic illness that is distinct from Kawasaki disease (16).

\section{Classification of MIS-C}

Recently, the CDC published initial findings of 570 children in the United States who were reported to fit its quite broad case definition of MIS-C. The study divided patients into three groups based on latent class analysis, a statistical modeling technique that divides cases into groups by underlying similarities. Patients in Class 1, which had the highest degree of organ involvement and higher prevalence of shock and lymphopenia, were judged to have little overlap with patients with Kawasaki disease. In contrast, patients in Class 3 more commonly met the criteria for Kawasaki disease (13). Patients in Class 2 had the most respiratory symptoms and highest prevalence of nasopharyngeal RT-PCR positivity for SARS-CoV-2, and likely had acute COVID-19. It should be remembered that acute COVID-19 can affect multiple organ systems, and that the presence of multiple organ involvement does not necessarily indicate a diagnosis of MIS-C (17). Some distinctive features of the three classes and Kawasaki disease are listed in Table 1. Based on this analysis, we conclude that Class 1 cases represent true MIS-C, and that many children with acute respiratory COVID-19 and with Kawasaki disease, two conditions that are distinct from MIS-C, were unintentionally included among reported cases because the CDC criteria are overly broad.

\section{Need for MIS-C pathogenesis studies}

The pathogenesis of MIS-C is unknown, and a postinfectious etiology has been hypothesized but not proven. SARS-CoV-2 antibodies arise in the second week after infection, but their presence does not indi- 
Table 1. Features of three nonoverlapping groups of children (Class 1, 2, and 3) reported to the CDC as MIS-C and their comparison with Kawasaki disease

Class 1 (classic MIS-C) ${ }^{A} \quad$ Class 2 (acute COVID-19) ${ }^{\mathrm{A}}$

\section{Class 3 (other conditions including Kawasaki disease) ${ }^{\mathrm{A}}$}

$\begin{array}{lccccc}\text { Median age in years } & 9 & 10 & 6 & & \\ \text { SARS-CoV-2 PCR', antibody } & 0.5 \% & 84 \% & 2 \% & \text { NA } & \text { Infrequent } \\ \text { Abdominal pain } & 80 \% & 49 \% & 54 \% & \text { Rare } \\ \text { Shock } & 76 \% & 28 \% & 1.6 \% & \text { Very rare }\end{array}$

${ }^{A}$ Reference 13. ${ }^{B}$ Reference 11. NA, not applicable.

cate resolution of infection. A recent study reported inefficient and reduced neutralizing antibody activity against SARSCoV-2 in patients with MIS-C, compared with adults with severe COVID-19 causing acute respiratory distress syndrome (ARDS) and adults who recovered from mild disease, suggesting a reduced protective serological response (18). The virus is generally not detected in the respiratory tract of patients with MIS-C (19), but other compartments such as the gastrointestinal tract have not yet been investigated.

Although the presence of SARS-CoV2-specific $\mathrm{T}$ cells in the peripheral blood of recovered and COVID-ARDS adult patients has been recently been reported (20), no such reports exist among children yet, and the biological significance of SARS-CoV-2-reactive $\mathrm{T}$ cells, whether protective or even detrimental, is still unclear (20). A direct effect of SARS-CoV2 Spike protein structure on immune activation has also been proposed (21). Indeed, recent data suggest that the SARS-CoV-2 Spike protein has a superantigen-like motif with sequence and structure homology to Staphylococcal enterotoxin B, which could mediate the hyperinflammation observed in MIS-C and in adults with severe COVID-19 and cytokine storm (21).

\section{The importance of distinguishing MIS-C and Kawasaki disease}

The etiology of Kawasaki disease remains unknown. Although the literature is rife with small unconfirmed studies purporting many different viruses are associated with Kawasaki disease, these appear to be coincidental and not causative. Indeed, a recent study using the very sensitive VirScan method, covering the complete reference protein sequences of known human viruses, failed to identify evidence of any recent viral infection that was more prevalent in children with Kawasaki disease than febrile childhood controls, including any coronavirus infection (22). A recent study from South Korea reported no temporal association of human coronavirus infection and incidence of Kawasaki disease at the national level (23). Recent research progress supports a presently unidentified so-called new respiratory RNA virus as the cause of the disease (24).

Some have suggested that MIS-C and Kawasaki disease cases should be included together in studies of pathogenesis of the two disorders (25). We take an alternate view. A variety of diverse infectious agents and noninfectious illnesses can cause inflammation affecting multiple organ systems. Although there are some basic commonalities in the immune response to diverse pathogens, the differences outnumber the similarities.

Combining diseases of clearly different etiologies together in studies of the pathogenesis will complicate efforts to understand the true spectrum of MIS-C and to identify its mechanisms and the optimal therapeutic targets. The argument that Kawasaki disease and MIS-C are similar because males are most often affected by both diseases fails to recognize the wellknown increased prevalence and severity of most pediatric infectious diseases among males (26). Similarly, the view that MIS-C and Kawasaki disease are part of a spectrum of the same disease because they both respond very well to intravenous immune globulin (IVIG) and steroids fails to recognize that several diseases with completely separate etiologies and different immunopathologies also respond to nonspecific antiinflammatory agents such as IVIG and steroids, including several autoimmune and rheumatologic diseases as well as TSS.

As noted in the CDC report, spread of the COVID-19 pandemic may increase the likelihood of patients with Kawasaki disease being misidentified as having MIS-C due to incidental findings of SARS-CoV-2 antibodies (13). Thus, we encourage careful distinction of cases of MIS-C-defined by prolonged fever, severe myocardial dysfunction, severe gastrointestinal symptoms, and lymphopenia with evidence of recent SARS-CoV-2 infection-from those in the differential diagnosis, including Kawasaki disease, prior to initiating any research investigations. This distinction is necessary to prevent incorrect conclusions being drawn due to the inclusion of unrelated diseases in the same research data set.

\section{Conclusions}

Elucidating the immune mechanisms of hyperinflammatory syndromes caused by SARS-CoV-2 infection, including MIS-C, will provide further insights for moretargeted treatment and potentially global prevention efforts. We believe that ongoing intense basic science investigations into the SARS-CoV-2 viral structures and the host immune response that may lead to the hyperinflammatory syndromes in both children (MIS-C) and adults with severe COVID-19 infection (cytokine storm) may provide additional clues to reveal the pathogenesis of these syndromes in the near future.

Address correspondence to: Moshe Arditi, 8700 Beverly Boulevard, Davis Building, Rooms D4024, 4025, 4027, Los Angeles, California 90048, USA. Phone: 310. 423.4471; Email: moshe.arditi@cshs.org. 
1. Belhadjer Z, et al. Acute heart failure in multisystem inflammatory syndrome in children (MIS-C) in the context of global SARS-CoV-2 pandemic. Circulation. 2020;142:429-436.

2. Ouldali N, et al. Emergence of Kawasaki disease related to SARS-CoV-2 infection in an epicentre of the French COVID-19 epidemic: a time-series analysis. Lancet Child Adolesc Health. 2020;4(9):662-668.

3. Rowley AH. Understanding SARS-CoV-2-related multisystem inflammatory syndrome in children. Nat Rev Immunol. 2020;20 (8):453-454.

4. Jhaveri S, et al. Longitudinal echocardiographic assessment of coronary arteries and left ventricular function following multisystem inflammatory syndrome in children (MIS-C) [published online August 4, 2020]. JPediatr. https://doi. org/10.1016/j.jpeds.2020.08.002.

5. Muniz JC, Dummer K, Gauvreau K, Colan SD, Fulton DR, Newburger JW. Coronary artery dimensions in febrile children without Kawasaki disease. Circ Cardiovasc Imaging. 2013;6(2):239-244.

6. Fox SE, Lameira FS, Rinker EB, Vander Heide RS. Cardiac endotheliitis and multisystem inflammatory syndrome after COVID-19 [published online July 29, 2020]. Ann Intern Med. https://doi.org/10.7326/L20-0882.

7. Fox SE, Akmatbekov A, Harbert JL, Li G, Quincy Brown J, Vander Heide RS. Pulmonary and cardiac pathology in African American patients with COVID-19: an autopsy series from New Orleans. Lancet Respir Med. 2020;8(7):681-686.

8. Dolhnikoff M, et al. SARS-CoV-2 in cardiac tissue of a child with COVID-19-related multisystem inflammatory syndrome [published online August 20, 2020]. Lancet Child Adolesc Health. https://doi. org/10.1016/S2352-4642(20)30257-1.
9. McCrindle BW, et al. Diagnosis, treatment, and long-term management of Kawasaki disease: a scientific statement for health professionals from the American Heart Association. Circulation. 2017;135(17):e927-e999.

10. Makino N, et al. Nationwide epidemiologic survey of Kawasaki disease in Japan, 2015-2016. Pediatr Int. 2019;61(4):397-403.

11. Holman RC, Curns AT, Belay ED, Steiner CA, Schonberger LB. Kawasaki syndrome hospitalizations in the United States, 1997 and 2000. Pediatrics. 2003;112(3 Pt 1):495-501.

12. Cameron SA, Carr M, Pahl E, DeMarais N, Shulman ST, Rowley AH. Coronary artery aneurysms are more severe in infants than in older children with Kawasaki disease. Arch Dis Child. 2019;104(5):451-455.

13. Godfred-Cato S, et al. COVID-19-associated multisystem inflammatory syndrome in children - United States, March-July 2020. MMWR Morb Mortal Wkly Rep. 2020;69(32):1074-1080.

14. Kim YJ, et al. Defining association between COVID-19 and the multisystem inflammatory syndrome in children through the pandemic. J Korean Med Sci. 2020;35(22):e204.

15. Xu S, Chen M, Weng J. COVID-19 and Kawasaki disease in children. Pharmacol Res. 2020;159:104951.

16. Carter MJ, et al. Peripheral immunophenotypes in children with multisystem inflammatory syndrome associated with SARS-CoV-2 infection [published online August 18, 2020]. Nat Med. https://doi.org/10.1038/s41591-020-1054-6.

17. Swann OV, et al. Clinical characteristics of children and young people admitted to hospital with covid-19 in United Kingdom: prospective multicentre observational cohort study. BMJ. 2020;370:m3249.

18. Weisberg SP, et al. Antibody responses to SARS $\mathrm{CoV} 2$ are distinct in children with MIS-C compared to adults with COVID-19. medRxiv. https:// doi.org/10.1101/2020.07.12.20151068. Published July 14, 2020. Accessed September 1, 2020.

19. Wölfel R, et al. Virological assessment of hospitalized patients with COVID-2019. Nature. 2020;581(7809):465-469.

20. Sette A, Crotty S. Pre-existing immunity to SARS-CoV-2: the knowns and unknowns. Nat Rev Immunol. 2020;20(8):457-458.

21. Cheng MH, et al. Superantigenic character of an insert unique to SARS-CoV-2 spike supported by skewed TCR repertoire in patients with hyperinflammation [published online September 28, 2020]. Proc Natl Acad Sci. https://doi. org/10.1073/pnas.2010722117.

22. Quiat D, et al. High-throughput screening of Kawasaki disease sera for antiviral antibodies [published online May 9, 2020]. J Infect Dis. https://doi.org/10.1093/infdis/jiaa253.

23. Choe SA, An HS, Choe YJ. No temporal association between human coronavirus and Kawasaki disease: National data from South Korea [published online August 14, 2020]. J Med Virol. https://doi.org/10.1002/jmv.26435.

24. Rowley AH, et al. A protein epitope targeted by the antibody response to Kawasaki disease. J Infect Dis. 2020;222(1):158-168.

25. Yeung RS, Ferguson PJ. Is multisystem inflammatory syndrome in children on the Kawasaki syndrome spectrum. JClin Invest. 2020;130(11):5681-5684.

26. Muenchhoff M, Goulder PJ. Sex differences in pediatric infectious diseases. J Infect Dis. 2014;209(Suppl 3):S120-S126. 